\title{
X-ray plasma ejections associated with coronal type II shocks
}

\author{
A. Shanmugaraju ${ }^{1}$, Y.-J. Moon ${ }^{2}$, Y.-H. Kim ${ }^{2}$, K.-S. Cho ${ }^{2}$, M. Dryer ${ }^{3}$, and S. Umapathy ${ }^{4}$ \\ 1 Department of Physics, Arul Anandar College, Karumathur - 625 514, Madurai (Dist.), India \\ e-mail: shanmugaraju_a@yahoo.com \\ 2 Korea Astronomy and Space science Institute (KASI), Whaamdong, Yuseong-gu, Daejeon 305-348, Korea \\ 3 NOAA Space Environment Center, Boulder, Colorado 80305, USA \\ 4 School of Physics, Madurai Kamaraj University, Madurai - 625 021, India \\ Received 6 February 2006 / Accepted 2 August 2006
}

ABSTRACT

\begin{abstract}
Context. Recent observations suggest that X-ray plasma ejections can drive coronal shocks and metric type IIs that are also generated in close association with the X-ray erupting features.

Aims. The physical relationship between the plasma ejections and metric type II radio bursts are studied by analyzing the characteristics of ejecta and type IIs.

Methods. We present the first comprehensive analysis of a set of 18 events of X-ray plasma ejections associated with coronal shocks inferred from metric type II radio bursts. For this study, we have utilized a list of $137 \mathrm{limb} \mathrm{X}$-ray plasma ejection events and multiwavelength observations from GOES X-ray, Yohkoh SXT, SOHO/LASCO, and SOHO/EIT.

Results. (i) type IIs are reported only for about $15 \%$ of the 137 limb ejections; (ii) there exists a close temporal relationship among the starting time of type IIs, the hard X-ray flare peak, and the ejecta time; (iii) there exist negative correlations between X-ray loop length and starting frequency, and between the ejecta height and starting frequency of type IIs; (iv) the type II formation height computed using the starting frequency and $1 \times$ Newkirk electron density model is in close association with or above the height of X-ray ejecta; and (v) while there is no correlation between the speeds of type II and ejecta, there seems to be a weak correlation between the speeds of type II and CME.

Conclusions. Though the results suggest that some type IIs are generated in close association with the X-ray erupting features, it is not likely that X-ray plasma ejections are the main drivers of all coronal shocks and metric type II radio bursts due to the absence of correlation between both speeds and mostly sub-Alfenic speeds of the ejections.
\end{abstract}

Key words. Sun: radio radiation - Sun: flares - Sun: coronal mass ejections (CMEs) - Sun: X-rays, gamma rays

\section{Introduction}

$\mathrm{X}$-ray plasma ejections are one of the most interesting phenomena observed by the Soft X-ray Telescope (SXT, Tsuneta et al. 1991) aboard Yohkoh. With high temporal coverage and resolution of Yohkoh, X-ray plasma ejections are often observed outside main flare loops in SXT images, especially around the impulsive phase of flares. Since the launch of Yohkoh in 1991, several studies of X-ray plasma ejections have been carried out, mainly focussing on the relation to magnetic reconnection (e.g., Shibata et al. 1995; Yokoyama \& Shibata 1996; Ohyama \& Shibata 1998; Karlicky 2004; Vrsnak et al. 2004). Type II bursts are the important probes of the magnetohydrodynamic (MHD) shocks generated by flares and/or coronal mass ejections (CMEs) in the solar corona (cf. Nelson \& Melrose 1985). Both the X-ray and radio observations are the necessary information regarding the eruption near the solar surface to study the physical relation between X-ray flares and type II shocks (Stewart 1977; Aurass et al. 1994). Despite the debate on the sources (flares or CMEs) of type IIs (Shanmugaraju et al. 2003a,b), recently, there have been reports (Gopalswamy et al. 1997, 1999, 2001) that proposed the possibility of X-ray ejecta as the driver of the coronal shock wave. In three different studies, they studied three events (on 1994 July 31, 1993 April 30, 1996 January 13) using both X-ray and radio observations. The latter workers identified the fact that X-ray ejecta in these events were fast enough to drive the coronal shock waves inferred from metric type II radio bursts.

On the other hand, the relation between early signatures in the type II radio spectrum and the features in the soft and hard X-ray imaging observations is established in a few independent events (Klassen et al. 1999a,b; Khan \& Aurass 2002; Klassen et al. 2003). That is, it has been found that the type II radio bursts are generated in association with and above the expanding soft X-ray loops.

In this regard, a comprehensive study on the X-ray imaging observations and metric type II radio observations is essential to understand the relation between them. We analyze a set of 18 events for which both the Yohkoh SXT observations and radio observations are available. Our main aim is to study the physical relationship between X-ray plasma ejections and metric type II bursts. For this study, we have utilized the multi-wavelength observations from GOES X-ray, Yohkoh SXT, SOHO/LASCO, and SOHO/EIT.

In the next section, we describe the data analysis. In Sect. 3, results and discussion are given. In Sect. 4, a brief conclusion is presented.

\section{Data analysis}

Out of 137 X-ray plasma ejections reported during the period 1999 April to 2001 March by Kim et al. (2005a), we have 
Table 1. Type II events and the corresponding X-ray flares and CMEs ${ }^{a}$.

\begin{tabular}{cccccccccccc}
\hline \hline DATE & \multicolumn{9}{c}{ TYPE II } & \multicolumn{1}{c}{ X-RAY FLARE } & \multicolumn{4}{c}{ CME } \\
& Start & End & Class & Start & Peak & End & Location & Time & PA & width & Speed \\
\hline 990403 & 2307 & 2317 & M4.3 & 2256 & 2310 & 2319 & N29E81 & 2347 & 74 & 156 & 1031 \\
990508 & 1452 & 1454 & M4.6 & 1422 & 1440 & 1501 & N23W75 & 1450 & 284 & 125 & 891 \\
990529 & 0311 & 0332 & M1.6 & 0304 & 0315 & 0327 & S23E63 & 0326 & 81 & 135 & 770 \\
990611 & 1115 & 1132 & C8.8 & 1107 & 1157 & 1231 & N42E90 & 1126 & 35 & 181 & 1442 \\
990716 & 1554 & 1603 & M3.1 & 1542 & 1550 & 1554 & N42W82 & 1630 & 301 & 111 & 815 \\
990725 & 1321 & 1329 & M2.4 & 1308 & 1338 & 1417 & N38W93 & 1331 & - & Halo & 1424 \\
990804 & 0551 & 0558 & M6.0 & 0545 & 0557 & 0614 & S16W64 & 0626 & 262 & 144 & 383 \\
991026 & 2130 & 2150 & M3.7 & 2109 & 2125 & 2137 & S15W88 & 2150 & 256 & 49 & 446 \\
991027 & 1329 & 1340 & M1.8 & 1324 & 1337 & 1401 & S16W87 & 1406 & 260 & 40 & 240 \\
000502 & 1448 & 1450 & M2.8 & 1442 & 1451 & 1456 & N21W70 & 1506 & 293 & 40 & 1476 \\
000618 & 0157 & 0212 & X1.0 & 0152 & 0159 & 0203 & N21W90 & 0220 & 307 & 132 & 368 \\
000623 & 1420 & 1449 & M3.0 & 1418 & 1431 & 1446 & N26W72 & 1454 & 293 & 103 & 997 \\
000727 & 0411 & 0415 & M2.4 & 0406 & 0410 & 0413 & N08W72 & - & - & - & - \\
000825 & 1435 & 1451 & M1.4 & 1421 & 1435 & 1446 & S15E67 & 1454 & 49 & 178 & 575 \\
001016 & 0655 & 0702 & M2.5 & 0640 & 0728 & 0911 & N03W90 & 0727 & 280 & 170 & 1165 \\
001206 & 2226 & 2255 & M1.6 & 2216 & 2230 & 2251 & S10W66 & 2326 & 263 & 62 & 316 \\
010306 & 1010 & 1011 & C6.7 & 1004 & 1013 & 1023 & S10W91 & 1050 & 266 & 80 & 474 \\
010324 & 0137 & 0149 & M1.2 & 0133 & 0138 & 0142 & S14W82 & 0206 & 275 & 61 & 408 \\
\hline
\end{tabular}

${ }^{a}$ If there is a blank, it means there is no report of the corresponding data.

collected a sample of 20 events for which type II radio bursts were reported in Solar Geophysical Data. There are no reports of type IIs for the remaining 117 events. From this list of 20 events, finally we obtained 18 events for which all the data were available. Table 1 gives the details of the 18 type II bursts associated with X-ray plasma ejections: date of observation is given in the first column; the data corresponding to the type IIs (start time and end time) are given in Cols. 2-3; X-ray flare information (class, start time, end time, and location) are given in Cols. 4-8; CME data (first appearance time in LASCO C2, width and position angle (PA) in degrees, and speed in $\mathrm{km} \mathrm{s}^{-1}$ ) are given in Cols. 9-12.

\section{Results and discussion}

Using the Yohkoh SXT and HXT flare mode observational data, Kim et al. (2005a) selected a sample of 137 X-ray plasma ejections and studied their association with CMEs. Also Kim et al. (2004) examined the morphology of these plasma ejections according to their shape and compared their physical properties. All the 137 events were limb events whose longitudes are larger than $60^{\circ}$. A subset of 43 among 137 ejections were found to be well observed, bright, and are mostly loop like ejections. In this paper, we have studied the association between X-ray plasma ejections and metric type II radio bursts. We have found that for about 15\% (20/137) ejections, type II radio bursts were reported by many observatories. Then we have studied the physical characteristics of $18 \mathrm{X}$-ray plasma ejections that are associated with the type II bursts. Most of these 18 events are in the subset of well observed bright events. Out of 18 events 12 are loop-like ejections, 5 are spray type ejections, and 1 is jet type ejection. These ejections do not correspond to the impulsive phase, unlike those studied by Shibata et al. (1995) and Nitta \& Akiyama (1999). Also the temporal relation of the ejection and the type II burst has varieties. In addition, the relation between the characteristics of plasma ejections and of type II bursts are analyzed.

Table 2 gives the details of the 18 type II radio bursts and their associated X-ray plasma ejections: date of observation is given in the first column; the data corresponding to the type IIs (start time, end time, starting frequency in $\mathrm{MHz}$, speed using $1 \times$ Newkirk model) are given in Cols. 2-5; X-ray plasma ejection information (ejecta time, speed, final ejecta height in solar radii, half loop length in the units of $10^{5} \mathrm{~km}$ and remarks about the times and resolution of the SXT images used to calculate the speed) are given in Cols. 6-10.

\subsection{X-ray plasma ejections}

Most of the ejecta associated with type II bursts have wellidentified X-ray features. For example, the X-ray imaging observations of two events are shown in Fig. 1 (1999 July 25, 13:09:06-13:13:42 UT) and Fig. 2 (1999 October 26, 21:12:52-21:23:03 UT). One can see the timing sequence of the expanding X-ray features in these images. Using the high time resolution flare mode data, the characteristics such as ejecta time and speed were already determined by Kim et al. (2005a). We have determined the characteristics such as final height and half loop length of these ejections. The ejecta height varies in the range 1.05-1.4 solar radii, and the half loop length varies in the range 1.0-3.60 $\times 10^{5} \mathrm{~km}$. Hard X-ray flares are reported for all these events and their peak times nearly coincide with the ejecta times.

\subsection{Radio emissions}

Metric type II radio bursts are reported mainly in a frequency range of $30-200 \mathrm{MHz}$ except for two events (000618, 001206). These type IIs were reported by several observatories in the world in Solar Geophysical Data (ftp: //ftp.ngdc.noaa.gov/STP/SOLAR_DATA). Each event was reported by many observatories. It should be noted that identification of a type II in a complex dynamic spectrum is difficult and depends on the subjectivity of an individual observer. If there were many reports for one event, we have considered the report of highest frequency because we wanted to estimate the type II formation height. Mainly, we have considered the information of type IIs (for example, starting frequency) identified and reported by them. An example of analysis of the dynamic 
Table 2. Basic information of Type II events and the corresponding X-ray plasma ejections ${ }^{a}$.

\begin{tabular}{|c|c|c|c|c|c|c|c|c|c|}
\hline \multirow[t]{2}{*}{ 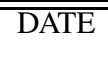 } & \multicolumn{4}{|c|}{ Type II } & \multicolumn{5}{|c|}{ Ejecta } \\
\hline & Start & End & Fs $(\mathrm{MHz})$ & Speed & Time & Speed & Height $\left(R_{\odot}\right)$ & Length $\left(10^{5} \mathrm{~km}\right)$ & Remarks \\
\hline 990403 & 2307 & 2317 & H180 & 594 & 2301 & 600 & - & $\mathrm{N}$ & $23: 05: 06-23: 05: 24(q)$ \\
\hline 990508 & 1452 & 1454 & H130 & 1749 & 1434 & 113 & 1.18 & 1.6 & $14: 34: 51-14: 35: 09$ (h) \\
\hline 990529 & 0311 & 0332 & H180 & 283 & 0308 & 928 & 1.215 & 2.0 & $03: 10: 00-03: 10: 18(q)$ \\
\hline 990611 & 1115 & 1132 & $170 \mathrm{U}$ & 354 & 1126 & 141 & 1.1 & $\mathrm{~N}$ & $11: 29: 07-11: 31: 15(\mathrm{~h})$ \\
\hline 990716 & 1554 & 1625 & $\mathrm{H} 170 \mathrm{U}$ & 668 & 1550 & 71 & 1.31 & $\mathrm{~N}$ & $15: 52: 52-15: 53: 10(q)$ \\
\hline 990725 & 1321 & 1329 & 80 & 952 & 1308 & 236 & 1.31 & 3.6 & $13: 14: 20-13: 14: 38(q)$ \\
\hline 990804 & 0551 & 0558 & $\mathrm{H} 220$ & 538 & 0558 & 378 & 1.234 & 2.6 & $06: 01: 32-06: 02: 44(q)$ \\
\hline 991026 & 2130 & 2150 & H150 & 322 & 2112 & 348 & 1.38 & 3.2 & $21: 23: 22-21: 23: 38(q)$ \\
\hline 991027 & 1329 & 1340 & 85 & 288 & 1327 & 104 & 1.19 & 2.1 & $13: 29: 48-13: 31: 24(\mathrm{~h})$ \\
\hline 000502 & 1448 & 1450 & 280 & 660 & 1454 & 174 & - & $\mathrm{N}$ & $15: 00: 54-15: 01: 08(q)$ \\
\hline 000618 & 0157 & 0212 & 650 & - & 0205 & 754 & 1.084 & $\mathrm{~N}$ & $02: 09: 40-02: 09: 52(\mathrm{~h})$ \\
\hline 000623 & 1420 & 1449 & 84 & 286 & 1420 & 2552 & 1.4 & 3.2 & $14: 24: 57-14: 25: 09(q)$ \\
\hline 000727 & 0411 & 0415 & H140 & 1325 & 0408 & 232 & 1.11 & 1.2 & $04: 12: 08-04: 12: 20(\mathrm{~h})$ \\
\hline 000825 & 1435 & 1451 & 164 & 425 & 1428 & 207 & 1.054 & $\mathrm{~N}$ & $14: 28: 46-14: 29: 18(f)$ \\
\hline 001016 & 0655 & 0702 & 68 & 1227 & 0643 & 621 & 1.37 & 2.8 & $06: 43: 29-06: 43: 57(q)$ \\
\hline 001206 & 2226 & 2255 & 800 & - & 2234 & 22 & 1.077 & 0.9 & $22: 36: 03-22: 36: 51(q)$ \\
\hline 010306 & 1010 & 1011 & H130 & 1399 & 1010 & 587 & 1.33 & 1.6 & $10: 12: 56-10: 13: 12(q)$ \\
\hline 010324 & 0137 & 0149 & $\mathrm{H} 230$ & 441 & 0137 & 373 & 1.14 & 1.0 & $01: 36: 40-01: 37: 36(\mathrm{~h})$ \\
\hline
\end{tabular}

$\mathrm{U}$ - the starting frequency is not clear. $\mathrm{H}$ - reported as harmonic frequency. $\mathrm{N}$ - may not be a loop type or not possible to measure. $\mathrm{q}$, h, $\mathrm{f}-\mathrm{corre}-$ sponds to quarter, half, full resolution of SXT images used to calculate speed. ${ }^{a}$ If there is a blank, it means the corresponding data are not possible to measure.
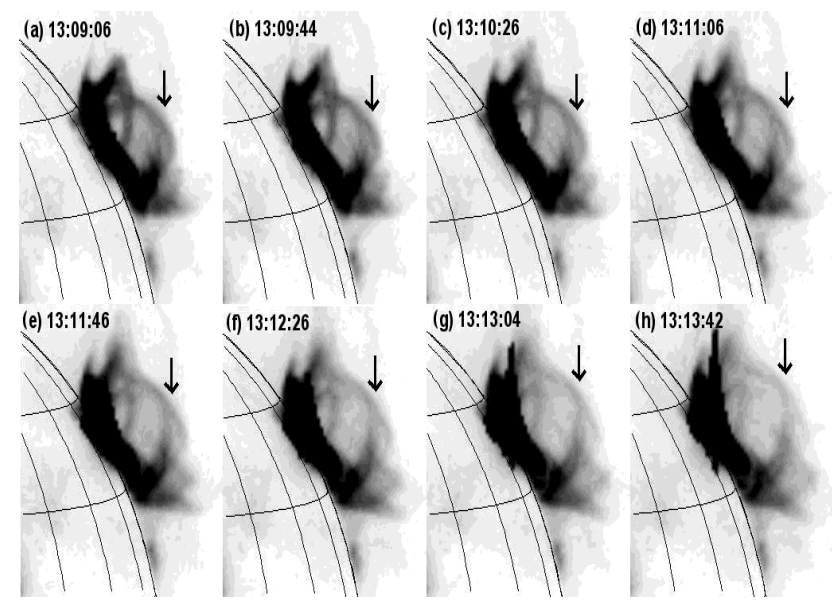

Fig. 1. A series of X-ray erupting features observed by Yohkoh SXT on 1999 July 25.

spectrum is given in Sect. 3.5. Observation of both the fundamental and harmonic bands were reported for many events. In these cases, we have considered the harmonic band for our analysis because the harmonic band is clearly visible than the fundamental in most of the dynamic spectra. In few cases in which the identification was not mentioned, we have considered them as harmonic because of the aforementioned reason. The starting frequency of harmonic band was divided by two in all the cases for the calculation of type II height and speed. Most of the type IIs start during the impulsive phase of the X-ray flares (between start and peak times of flares). Out of 18 events, there are reports (http://sunkl.asu.cas.cz/ radio/info.htm) of decimetric radio emissions for 10 events within the frequency range 800-4500 MHz. These decimetric emissions started after the start of the X-ray flare and ended before the start of metric radio emissions. The simultaneous occurrence of these emissions is in agreement with the propagation of eruptive features from the solar surface towards the outer corona.

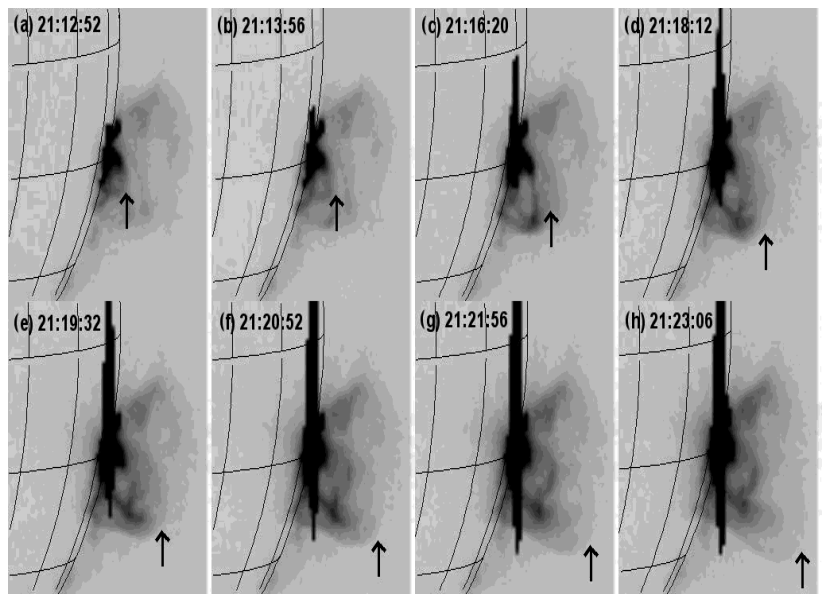

Fig. 2. A series of X-ray erupting features observed by Yohkoh SXT on 1999 October 26.

\subsection{Relation between type II radio bursts and plasma ejections}

The start times of type II bursts are compared with the other associated X-ray features. We found the time delay between the following events: type II start - hard X-ray flare peak time; type II start - ejecta time; and type II start - soft X-ray flare peak time. The delays are found to be within -20 to $20 \mathrm{~min}$ as shown in Figs. 3a,b, and d. These results imply that all the above phenomena occurred very closely in time. More importantly, the hard X-ray peak and ejecta time correspond well with the type II start as seen in Figs. $3 a$ and b. On the other hand, the time delay between type II start - X-ray flare start lies between 0 to 20 min (see Fig. 3c).

Further, vis-a-vis the relation between the flare rise time and starting frequency, it was suggested by Vrsnak (2001) that, if the flare rise time is small, the starting frequency should be high (Shanmugaraju et al. 2003a). Regarding this study, we considered only events for which loop length measurements are possible (see Table 2) and starting frequency is below $250 \mathrm{MHz}$. 

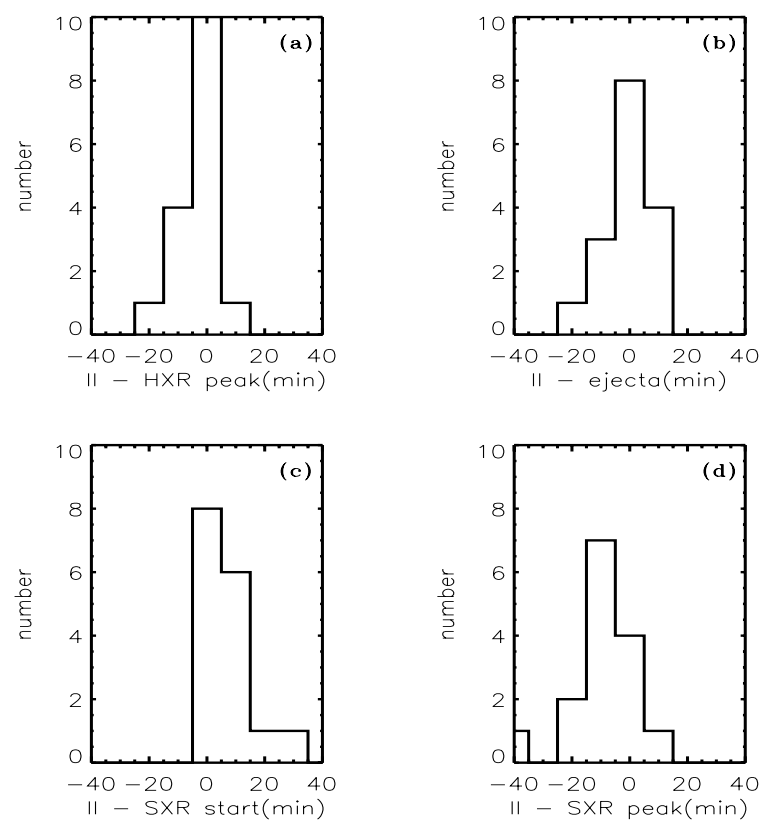

Fig. 3. Distributions of the delay time a) type II start - hard X-ray flare peak time; b) type II start - ejecta time; c) type II start - soft X-ray flare start; d) type II start - X-ray flare peak.
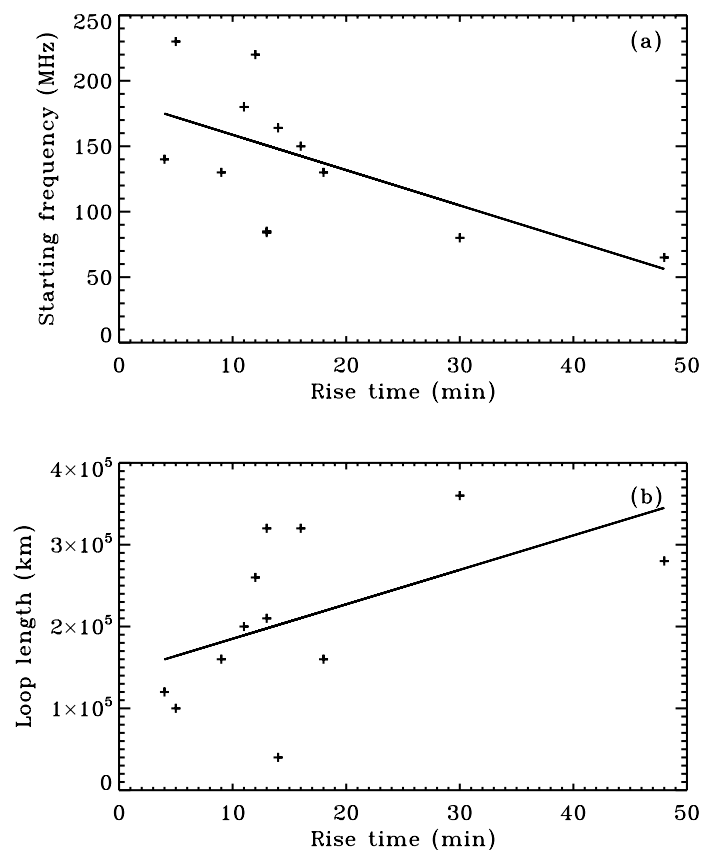

Fig. 4. Correlation between a) flare rise time and starting frequency, and b) flare rise time and loop length.

As a result we got only 12 events, and only these events are plotted in Figs. 4 and 5. The result of Vrsnak (2001) is clearly seen in our sample as shown in Fig. 4a. Also a weak positive correlation $(50 \%)$ is found (Fig. 4b) between the flare rise time ( $T_{\text {rise }}$ in min) and half loop length ( $L$ in $\mathrm{km})$.

$L=4210 \times T_{\text {rise }}+1.43 \times 10^{5}$.

These results imply that there should be some relation between starting frequency of type II bursts and X-ray loop length. Motivated by these results, we studied the correlation between ejecta height and starting frequency (Fig. 5a), and, loop length and starting frequency (Fig. 5b). As seen in these figures, there
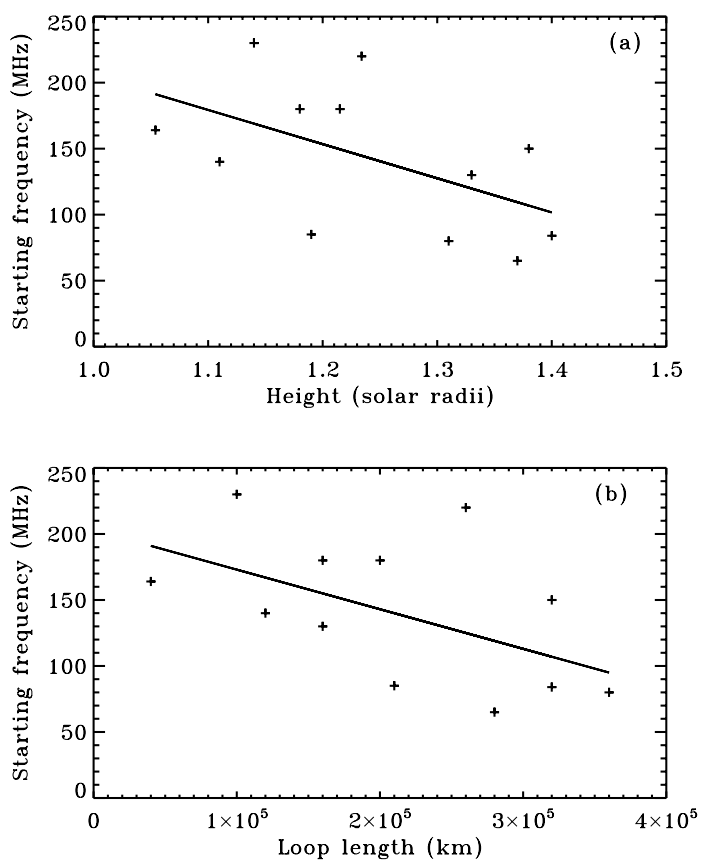

Fig. 5. Relationship between a) ejecta height and starting frequency, and b) loop length and starting frequency.

exists an inverse relation between the starting frequency and ejecta height or loop length. That is, the greater the X-ray loop length, the smaller the starting frequency of type IIs. In the solar corona, the electron density decreases with respect to altitude (Newkirk 1961),

$N_{\mathrm{e}}=m \times N_{\mathrm{o}} \times 10^{4.32\left(R_{\odot} / R\right)}$,

where $N_{\mathrm{o}}=4.2 \times 10^{4} \mathrm{~cm}^{-3}, m$ is the density multiplier, and $R_{\odot}$ is one solar radius.

The plasma frequency is related with electron density as

$f_{\mathrm{p}}=9000 \sqrt{N_{\mathrm{e}}} \mathrm{Hz}$.

It is widely accepted that the observed radio emission is equal to the plasma frequency in the corona. Hence, greater loop length or higher ejection height means lower density and, so, lower plasma frequency. The above result is in agreement with the recent results of Klassen et al. (2003) that the type II onset is associated with the expanding soft X-ray loops.

Using the relations, Eqs. (2) and (3) between frequency and density, we can derive a relation for type II formation height as,

$H_{\mathrm{II}}=\frac{2.16 R_{\odot}}{\log \left(f_{p}\right)-\log \left(1845 \times 10^{3} \times \sqrt{m}\right)}$.

\subsection{Relation between type II, CME and ejecta heights}

We have computed the type II formation height using the starting frequency for $1 \times$ Newkirk model. For two events, 000618 and 001206 , the type II starting frequency were reported unusually high (650 MHz and $800 \mathrm{MHz}$, respectively). We neglected these two events for this analysis. The type II formation heights are plotted against the ejecta heights in Fig. 6a. As seen in this figure, the ejecta height range $\left(1.05-1.4 R_{\odot}\right)$ lies within the type II formation height range $\left(1.05-1.7 R_{\odot}\right)$ calculated using $1 \times$ Newkirk model. In addition, the type II formation height is plotted against the CME height as shown in Fig. 6b. The height 

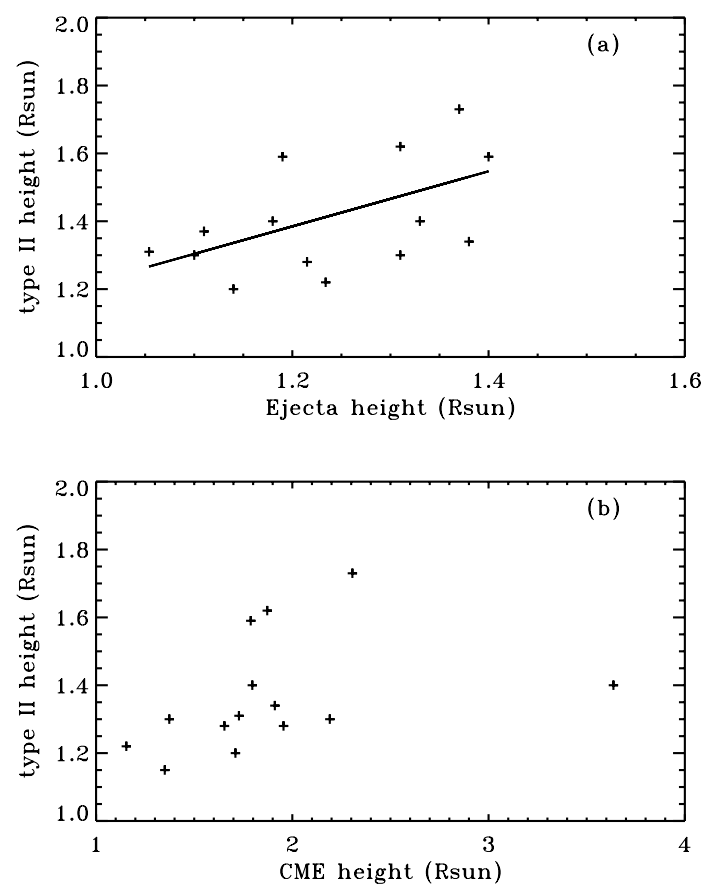

Fig. 6. Relationship between a) ejecta height and type II formation height, and b) CME height at the time of type II start and type II formation height (type II formation height is determined using $1 \times$ Newkirk model).

of the CME at the time of type II start is determined using the relation,

$H_{\mathrm{CME}}=H_{\mathrm{o}}-V_{\mathrm{CME}} \times \delta t$,

where $H_{\mathrm{o}}$ is the height at which the CME was first detected in LASCO C2, and $\delta t=T_{\mathrm{CME}}-T_{\text {type II }}$, is the time difference between the time of first observation of CME in LASCO C2 and the time of type II start. It is interesting to note the weak correlations between the type II formation height with both the ejecta height and CME height.

\subsection{Relation between type II, CME and ejecta speeds}

As given in Table 1, there are CME reports for all the events except one. The minimum width of the CME is $40^{\circ}$. The CME speeds are within $316-1476 \mathrm{~km} \mathrm{~s}^{-1}$ whereas the ejecta speeds are within $22-2552 \mathrm{~km} \mathrm{~s}^{-1}$. Only 6 out of 18 ejecta have speeds greater than $400 \mathrm{~km} \mathrm{~s}^{-1}$. In order to study the relation between the speeds of type IIs, ejecta and CMEs, we have computed the speed of type II shocks using the relation (Cho et al. 2005),

$V_{\mathrm{II}}=\frac{-2 R^{2} \mathrm{~d} f / \mathrm{d} t}{\ln 10 \times 4.32 R_{\odot} f_{\mathrm{p}}}$.

The type II speeds are calculated in the following way. First, the type II formation height is evaluated from Eq. (4) using the fundamental starting plasma frequency and $1 \times$ Newkirk model. For example, the type II event on 990403 is shown in Fig. 7. Here, the harmonic band (reported by Culgoora in the frequency range 180-40 MHz during 2307-2317 UT) is clearer than the fundamental. The type II formation height corresponding to the fundamental starting plasma frequency of $90 \mathrm{MHz}$ is found to be $1.28 R_{\odot}$. Second, an average drift rate is determined from the starting and ending frequencies, and the time duration of type IIs $\left(\mathrm{d} f / \mathrm{d} t=\left(f_{\mathrm{p}}-f_{\mathrm{e}}\right) / T_{\mathrm{d}}\right)$. For example, an average drift

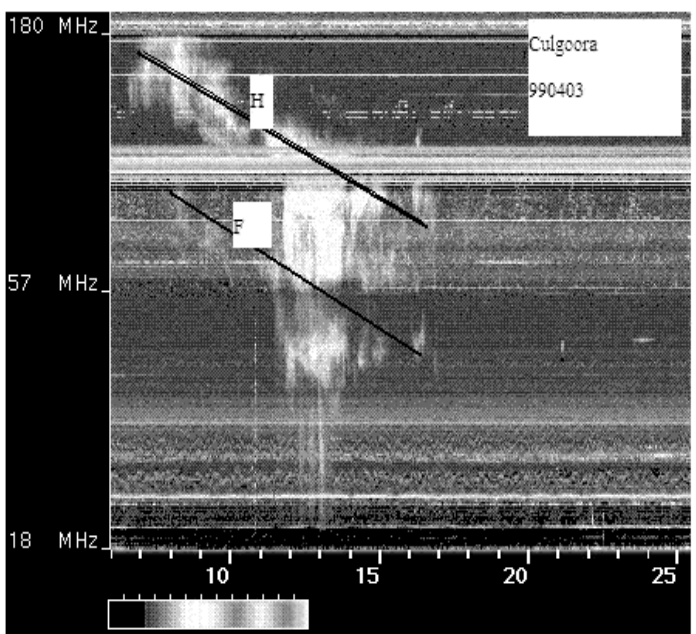

Fig. 7. An example of a type II burst observed by Culgoora on 1999 April 3 during 2307-2317 UT. The fundamental and harmnonic are marked as $\mathrm{F}$ and $\mathrm{H}$, respectively.
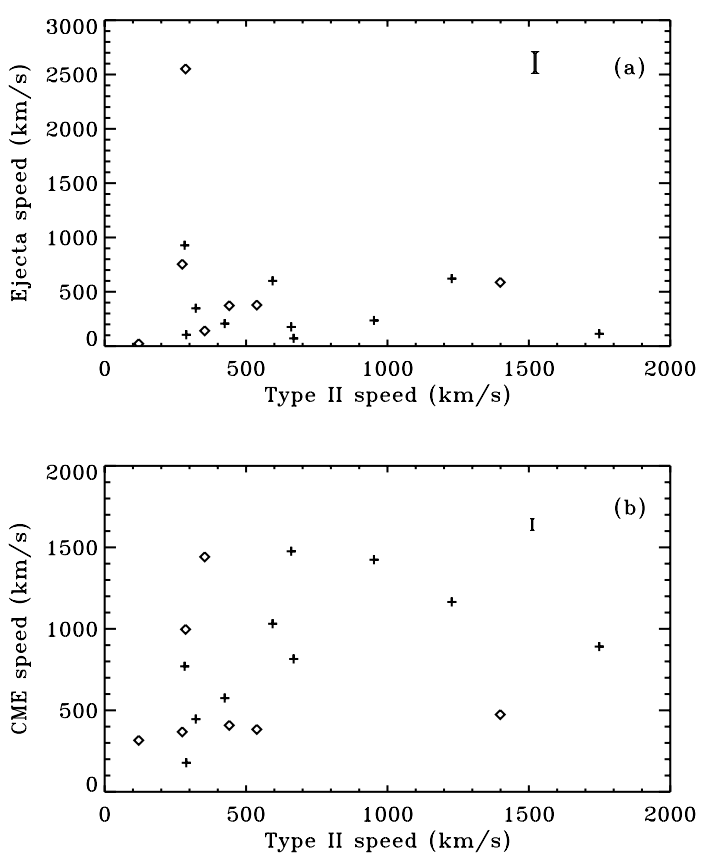

Fig. 8. a) Comparison between type II speed and ejecta speed. The vertical error bar in right upper corner indicates an error of $150 \mathrm{~km} \mathrm{~s}^{-1}$ for quarter-resolution images of Yohkoh flare mode data. b) Comparison between type II speed and CME speed (type II speeds are calculated using $1 \times$ Newkirk model). The vertical error bar in right upper corner indicates an error of $56 \mathrm{~km} \mathrm{~s}^{-1}$ for a height error of 23 arcsec in SOHO/LASCO images. (The seven events that were observed during type II emission time are marked with diamond symbols.)

rate of $0.23 \mathrm{MHz} \mathrm{s}^{-1}$ is estimated for the same event in the above frequency range and time duration. Third, the type II speeds are estimated utilizing these values in Eq. (6). It is estimated as $594 \mathrm{~km} \mathrm{~s}^{-1}$ for the above event.

They are compared with both the ejecta speed and CME speed (Figs. 8a and b). As seen in these figures, while there is no correlation between type II and ejecta speeds, a weak correlation $(r=0.3)$ is found between type II and CME speeds. Also, we found no correlation between ejecta and CME speeds in this subset of 18 events as recently pointed out by Kim et al. (2005b) for all 137 events. As discussed by Kim et al. (2005b), 
major accelerations of X-ray plasma ejections usually occur in the hard X-ray flaring time. Thus, it is not likely that that such a "no correlation" situation can become a "good correlation". The absence of correlation between type II speed and ejecta speed may be partially attributed to several assumptions in estimating the type II speeds and to the fact that the X-ray plasma ejections (XPEs) are the erupting features in the low corona.

It is also important to mention the significant uncertainties in the measurements, especially the speeds of type IIs, ejecta and CMEs. First, it appears that the measured speed of an ejection tends to be low when it is observed only late in the flare (For example, the events on 990508, 990716). Also, it appears that one sees only internal structures of the ejection in the 1999 July 25 event (Fig. 1) with the front possibly having been formed at higher altitudes. This may be a reason the speed of this event is low, although well measured. Even after removing these events, we found no correlation between the type II and ejecta speeds. In the case of X-ray plasma ejections, we used half and quarter resolution images of Yohkoh flare mode data. After several trial measurements, we estimated the height error to be about $5^{\prime \prime}$ for quarter-resolution image and about $2.5^{\prime \prime}$ for half-resolution image. Since the time interval between successive images that we considered is about $50 \mathrm{~s}$, the speed errors can be estimated as about $150 \mathrm{~km} \mathrm{~s}^{-1}$ for quarter-resolution and about $75 \mathrm{~km} \mathrm{~s}^{-1}$ for half-resolution, respectively.

Second, the type II speeds are quite different depending on the density model. For example, when we utilized a different density multiplier $(m=2)$, we found an average of $20 \%$ deviation between the speeds corresponding to two different density models $(1 \times$ Newkirk and $2 \times$ Newkirk). An average type II speed error in $\mathrm{km} \mathrm{s}^{-1}$ would be $\Delta V_{\mathrm{II}}=V_{\mathrm{II}} \times 0.20$. That is, the speed error is large for high speed events. In Fig. 8, there are only two type II events having speeds greater than $1000 \mathrm{~km} \mathrm{~s}^{-1}$. The measurement error in frequency drift is also one of the effects causing uncertainty of the type II speed.

Third, the error in estimating CME speed is as follows. LASCO C2 covers the range 2-6 solar radii with a resolution of about $23^{\prime \prime}$ and a pixel size of $11.9^{\prime \prime}$. If we adopt that the height error is 23 arcsecs, then the speed error in $\mathrm{km} \mathrm{s}^{-1}$ would be $\Delta V_{\mathrm{CME}}=2 \times 23 \times 725 / t_{\mathrm{c}}$, where $t_{\mathrm{c}}$ is the time cadence in s. Assuming an average time cadence of LASCO C2 images as $10 \mathrm{~min}$, the speed error can be determined as $56 \mathrm{~km} \mathrm{~s}^{-1}$, which is roughly less than $10 \%$ of the mean speed of CMEs associated with type IIs (704 $\mathrm{km} \mathrm{s}^{-1}$, Shanmugaraju et al. 2003b). Also one has to keep in mind the error due to the projection effect of CMEs. Besides, CME speed extrapolation from LASCO field of view to type II formation height also has an error.

\subsection{XPEs as the sources of type II radio bursts}

Although the flares and CMEs are believed to be the sources of coronal shocks, recent investigations (Gopalswamy et al. 1997, 1999, 2001; Klassen et al. 1999a,b; Khan \& Aurass 2002; Klassen et al. 2003) suggested that X-ray plasma ejections may also be considered as the drivers of coronal shocks and hence the metric type II radio bursts. For example, the three X-ray plasma ejections studied by Gopalswamy et al. $(1997,1999,2001)$ had speeds $900 \mathrm{~km} \mathrm{~s}^{-1}, 670 \mathrm{~km} \mathrm{~s}^{-1}$ and $206 \mathrm{~km} \mathrm{~s}^{-1}$, respectively. Though the third ejecta had a lower limit speed of $206 \mathrm{~km} \mathrm{~s}^{-1}$, all of the three ejecta were found to have good correspondence with type II sources, and, so, they suggested that these ejecta were the drivers of the respective coronal shocks. In the case of Khan \& Aurass (2002), the soft X-ray disturbance was observed to travel with a speed of about $546 \mathrm{~km} \mathrm{~s}^{-1}$, and the type II source was

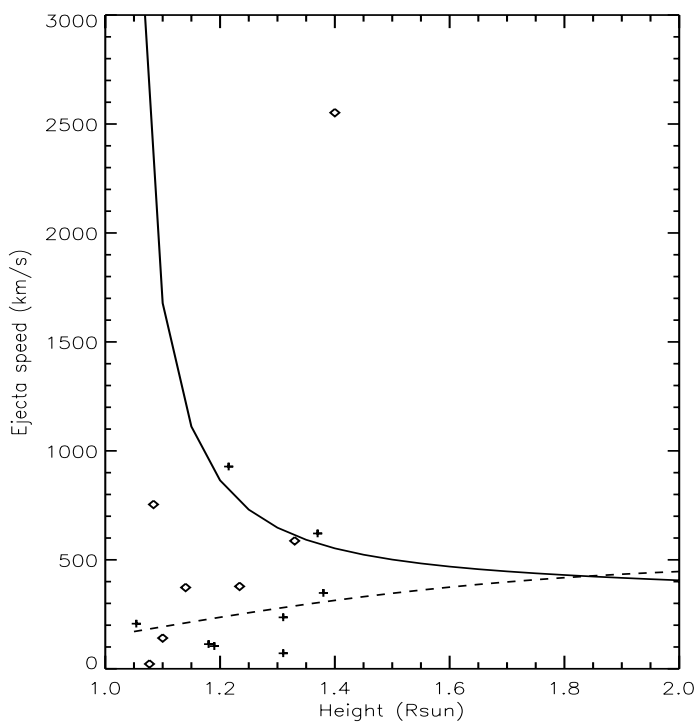

Fig. 9. Ejecta speed vs. ejecta height measured using Yokhoh SXT images. Alfven speeds are computed using $1 \times$ Newkirk model for both active region magnetic field model (solid line) and quiet Sun magnetic field model (dashed line). The seven events that were observed during type II emission time are marked with diamond symbols.

found to be located close to the soft X-ray disturbance, and it also showed motions consistent with soft X-ray motions. In support of these results, Klassen et al. (2003) identified the type II precursor as a signature of the reconnection process above the soft X-ray loops which later generated the type II burst.

On the other hand, the XPEs can generate shocks if the speed of XPE is greater than the local Alfvenic speed. Figure 8 shows the ejecta speed as a function of height. As mentioned already in our sample also, there are 6 ejecta having speeds greater than $400 \mathrm{~km} \mathrm{~s}^{-1}$. In this figure, Alfven speeds are also plotted for comparison. They were computed using $1 \times$ Newkirk model for both active region magnetic field model (solid line) and quiet Sun magnetic field model (dashed line) using the following relation:

$V_{\mathrm{A}}=2 \times 10^{11} B N_{\mathrm{e}}^{-1 / 2} \mathrm{~cm} \mathrm{~s}^{-1}$

with active region magnetic field model (Dulk \& McLean 1978),

$B_{\text {active }}=0.5\left(R / R_{\odot}-1\right)^{-1.5}$ Gauss

and the quiet Sun magnetic field model (Mann et al. 1999),

$B_{\text {quiet }}=2.2\left(R_{\odot} / R\right)^{2}$ Gauss.

As seen in the figure, most of the ejecta speeds are sub-Alfvenic.

As found from the present study, three phenomena (hard $\mathrm{X}$-ray, type II time, XPE) are highly associated. Thus we may expect some correlation between physical parameters of XPEs and type II events though XPE is not a driver of type II shock. More important constraints are (1) the comparison between type II speed and ejecta speed and (2) whether ejecta speed is super-Alfvenic or not. Figures 8 and 9 do not support the XPE origin. We also examined in more detail the following seven events (990611, 990804, 000618, 000623, 001206, 010306, 010324) since the ejecta were observed during type II emission time. In this case, we expect some correlation between ejecta speed and type II speed without any extrapolation if the XPEs are the drivers of type II bursts. For all the seven events, there is no correlation between ejecta speed and type II speed. 
Even though we can not rule out a possibility that some type IIs are generated by XPEs, it is not likely that the XPEs are the main driver of all type II bursts. On the other hand, the relationship between type IIs and CMEs seems to be better from the points (i) weak correlation between type II and CME speeds as in Fig. 8b, and (ii) super-Alfvenic speeds of many CMEs as in Table 2. Regarding this issue, we note that some of the recent works favor the CME origin (for example, Cliver et al. 1999; Shanmugaraju et al. 2006; and Cho et al. 2005). Cho et al. (2005) found that a large fraction (81\%) of the type IIs have temporal and spatial association with CMEs, and the association increases as their source position approaches to the limb. In addition, Shanmugaraju et al. (2006) investigated the origin of coronal shocks without mass ejections observed during April 1997-December 2000. By analyzing temporal and spatial relationship between flares, type IIs and CMEs of limb events (longitude $\geq 45^{\circ}$ ), they found that there were no coronal shocks without mass ejections except two cases.

\section{Conclusion}

We have made the first comprehensive analysis of a set of 18 events of X-ray plasma ejections observed by the space-based Yokhoh satellite (during the period 1999 April to $2001 \mathrm{March}$ ). These events are associated with coronal shocks inferred from metric type II radio bursts observed by many ground-based radio spectrographs. For this study, we have utilized the list of 137 limb X-ray plasma ejection events reported by Kim et al. (2005a). The physical relationship between the plasma ejections and type II radio bursts is investigated by analyzing the characteristics of ejecta and type IIs. The main results obtained from this study are: (i) the X-ray ejections compiled by Kim et al. (2005a) are poorly correlated with metric type II bursts (18/137); (ii) there exists a close temporal relationship between the starting time of type IIs with the hard X-ray flare peak and ejecta time; (iii) there exist negative correlations between $\mathrm{X}$-ray loop length and starting frequency and also between the ejecta height and starting frequency of type IIs; (iv) the type II formation height computed using the starting frequency and $1 \times$ Newkirk electron density model is in close association with or above the height of X-ray ejecta; and (v) while there is no correlation between the type II speeds and ejecta speeds, there seems to be a weak correlation between the speeds of type IIs and CMEs. These results suggest that, while there are examples in which the observed X-ray ejection seems to be linked with a type II burst, the absence of correlation between the speeds of ejecta and type IIs on the whole, and the sub-Alfvenic speeds of the ejections are not in the favor of the ejecta to be the main driver of all coronal shocks. Finally, we suggest that it is necessary to simultaneously examine possible candidates (flare, CME, and X-ray plasma ejections) at the type II burst time in order to draw a more definite conclusion on the origin of type II coronal shock.

Acknowledgements. We thank the referee for his/her constructive comments on the manuscript. This work has been supported by the MOST grants (M1-010400-0059 and M1-0407-00-0001) and the Korea Research Foundation (KRF2005-070-C00059) of the Korean government. M.D. has been partially supported by a US DoD/USAF/UPOS project (via the University of Alaska at Fairbanks, Geophysical Institute) and by a NASA/LWS contract to Exploration Physics International, Inc. The CME catalog we have used is generated and maintained by the Center for Solar Physics and Space Weather, The Catholic University of America in cooperation with the Naval Research Laboratory and NASA. SOHO is a project of international cooperation between ESA and NASA.

\section{References}

Aurass, H., Klein, K. L., \& Mann, G. 1994, ESA SP, 373, 95

Cho, K.-S., Moon, Y.-J., Dryer, M., et al. 2005, J. Geophys. Res., 110 (A12), A12101

Cliver, E. W., Webb, D. F., \& Howard, R. A. 1999, Sol. Phys., 187, 89

Dulk, G. A., \& McLean, D. I. 1978, Sol. Phys., 57, 279

Gopalswamy, N., Kundu, M. R., Manoharan, P. K., et al. 1997, ApJ, 486, 1036

Gopalswamy, N., Nitta, N., Manoharan, P. K., Raoult, A., \& Pick, M. 1999, A\&A, 347, 684

Gopalswamy, N., St, Cyr, O. C., Kaiser, M. L., \& Yashiro, S. 2001, Sol. Phys., 203, 149

Karlicky, M. 2004, A\&A, 417, 325

Khan, J. I., \& Aurass, H. 2002, A\&A, 383, 1018

Kim, Y.-H., Moon, Y.-J., Cho, K.-S., Bong, S.-C., \& Park, Y. D. 2004, J. Korean Astron. Soc., 37, 171

Kim, Y.-H., Moon, Y.-J., Cho, K.-S., Kim, K.-S., \& Park, Y. D. 2005a, ApJ, 622, 1240

Kim, Y.-H., Moon, Y.-J., Cho, K.-S., Bong, S.-C. \& Park, Y. D. 2005b, ApJ, 635, 1291

Klassen, A., Aurass, H., Klein, K.-L., Hofmann, A., \& Mann, G. 1999, A\&A, 343,287

Klassen, A., Pohjolainen, S., \& Klein, K.-L. 2003, Sol. Phys., 218, 197

Mann, G., Aurass, H., Klassen, A., Estel, C., \& Thompson, B. J. 1999, in Proc. 8th SOHO Workshop, ed. B. Kaldeich-Schurmann, ESA SP-466, 477

Nelson, G. J., \& Melrose, D. B. 1985, in Solar Radiophysics, ed. D. J. McLean, \& N. R.Labrum (Cambridge: Cambridge University Press), 350

Newkirk, G. A. 1961, ApJ, 133, 983

Nitta, N., \& Akiyama, S. 1999, ApJ, 525, L57

Ohyama, M., \& Shibata, K. 1998, ApJ, 499, 934

Shanmugaraju, A., Moon, Y.-J., Dryer, M., \& Umapathy, S. 2003a, Sol. Phys., 215,161

Shanmugaraju, A., Moon, Y.-J., Dryer, M., \& Umapathy, S. 2003b, Sol. Phys., 217,301

Shanmugaraju, A., Moon, Y.-J., Cho, K.-S., Dryer, M., \& Umapathy, S. 2006, Sol. Phys., 233, 117

Shibata, K. 1996, Adv. Space. Res., 17(4/5), 9

Shibata, K., Masuda, S., Shimojo, M., et al. 1995, ApJ, 451, L83

Stewart, R. T. 1977, Proc. Astron. Soc. Aust., 3, 157

Tsuneta, S., Acton, L., Bruner, M., et al. 1991, Sol. Phys., 136, 37

Vrsnak, B. 2001, J. Geophys. Res., 106(A11), 25291

Vrsnak, B., Maricic, D., Stanger, L., \& Veronig, A. 2004, Sol. Phys., 225, 355

Yokoyama, T., \& Shibata, K. 1996, PASJ, 48, 353 